\title{
Estimation of mutation rates in cultured mammalian cells
}

\author{
I-Chian Li ${ }^{1}$, Jiliang Fu ${ }^{1, *}$, Yung-Tai Hung ${ }^{2}$ and E.H.Y. Chu ${ }^{1}$ \\ ' Department of Human Genetics, Medical School, and ${ }^{2}$ Department of Biostatistics, School of Public \\ Health, University of Michigan, Ann Arbor, MI 48109 (U.S.A.)
}

(Received 19 Augst 1982)

(Revision received 26 May 1983)

(Accepted 31 May 1983)

The factors that affect reliable estimations of mutation rates $(\mu)$ in cultured mammalian somatic cell populations by fluctuation analysis are studied experimentally and statistically. We analyze the differential effect of the final cell population size in each culture $\left(N_{t}\right)$ and the number of parallel cultures $(C)$ on the variation in the rate estimates $(\hat{\mu})$ inferred from the $P_{0}$ method. The analysis can be made after the derivation of the variance of $\hat{\mu}$, which is a measure of variation of $\hat{\mu}$ for a given combination of $N_{\mathrm{t}}$ and $C$ in a number of repeat experiments. The variance of $\hat{\mu}$ is inversely proportional to $C$ and to the square of $N_{\mathrm{t}}$. $N_{\mathrm{t}}$ determines the probability of occurrence of mutations in a cell culture. By influencing the size of $P_{0}, N_{\mathrm{t}}$ also determines whether a rate estimate is obtainable from the experiment. Since $P_{\mathrm{o}}$ is estimated from the fraction of cultures containing no mutation in a set of $C$ cultures, $C$ becomes a determining factor for the accuracy of $\hat{\mu}$. The rate estimated from $\hat{P}_{0}$ is biased, but the bias is in general 2 orders of magnitude smaller than $\hat{\mu}$. By the selection of an appropriate combination of $N_{\mathrm{t}}$ and $C$ for the experiment, this bias can be reduced even further.

Based on the notion of comparing two proportions, we propose a test statistic and have applied it to experimental results for a test of equality of mutation rates in different cell lines. This development places the comparison of mutation rates on a statistical basis.

Since the publication of Luria and Delbrück's paper [19] on the distribution of the number of mutant colonies in a series of parallel cultures, fluctuation analysis has been widely applied as a method for the determination of mutation rates $(\mu)$ in cell populations. The analysis has since been further developed methodologically by Lea and Coulson [15], Newcombe [22], and Armitage [2,3] and modified theoreti-

\footnotetext{
* Visiting scholar from the School of Public Health, Sichuan Medical College, Chengdu, Sichuan, China.
} 
cally by Kondo [14]. Although other methods [22-24] are available, fluctuation analysis has reamained the most popular method for the estimation of $\mu$.

In mammalian cell lines grown in vitro, $\mu$ values estimated by fluctuation analysis vary considerably. In the data compiled by Morrow [21], for instance, the rate estimates $(\hat{\mu})$ for the same genetic marker in a cell line with the same designation but grown in different laboratories vary by as much as 2000 -fold. A variety of factors may be responsible for this discrepancy. First, unidentified differential mutabilities among the sub-lines studied may contribute in part to the variation. Second, the procedures of cell culture such as dissociation of cells by trypsinization, the cell number per culture dish, the growth medium, and the concentration of selective agent may influence the recovery of mutants [10]. Third, a source of variability of $\hat{\mu}$ may come from the phenotypic lag and possible selective disadvantage of the mutant cells, as discussed by Koch [13]. Finally, the number of parallel cultures $(C)$ and the final cell population size $\left(N_{1}\right)$ of any one culture may contribute greatly to the variation in the estimated mutation rate [11]. Taking several published reports as examples, $C$ varies from 6 [26] to 120 [25], and $N_{\mathrm{t}}$ varies from $10^{4}$ to $10^{8}$ cells in different experiments $[20,25,26]$. It is therefore difficult to know which of the published mutation rates are dependable and whether a rate comparison between cell types, genetic markers or experiments is at all possible.

The factors that most affect the estimation of $\mu$ by fluctuation analysis are $N_{\mathrm{t}}$ and $C$. The purposes of this paper are: (1) to analyze experimentally and statistically the interaction between the two parameters, (2) to design a practical experimental scheme by which reliable estimation of $\mu$ may be assured, and (3) to find a test statistic for a comparison of mutation rates in different cell lines. A preliminary account of this work has been published elsewhere [16].

\section{Results}

Statistical consideration of the effects of $N_{t}$ and $C$ on the estimation of $\mu$

According to Luria and Delbrück [19], the probability, $P_{0}$, that no mutation has occurred in an experiment can be expressed as:

$P_{\mathrm{o}}=\mathrm{e}^{-\mu N_{\mathrm{1}}}$

or

$\mu=\left(-\ln P_{0}\right) / N_{\mathrm{t}}$

Since $\mu$ is a constant, $P_{0}$ becomes a function of $N_{\mathrm{t}}$; i.e., $N_{\mathrm{t}}$ determines whether there will be some mutations detectable in any particular culture. In practice, $P_{0}$ is derived from the number of cultures $(X)$ containing no mutations in a set of $C$ sib cultures, i.e., $\hat{P}_{0}=X / C$. Hence $C$ determines in part whether an appropriate $P_{0}$ is obtainable. We have proposed elsewhere [11] that at least $X=10$ sib cultures containing mutations are needed for a reliable estimation of $\mu$.

The expected value of $\hat{P}_{0}$ is equal to $P_{0} ; \hat{P}_{0}$ is therefore an unbiased estimate of $P_{0}$. 
From Eqn. 1b:

$\hat{\mu}=\left(-\ln \hat{P}_{0}\right) / N_{\mathrm{t}}$

The expectation $(E)$ and variance (var) of $\hat{\mu}$ can be derived by applying a Taylor series expansion to Eqn. 2:

$E(\hat{\mu}) \doteq \mu+\frac{1-P_{0}}{2 C N_{\mathrm{t}} P_{0}}=\mu+$ bias

and

$\operatorname{var}(\hat{\mu}) \doteq \frac{1-P_{0}}{C N_{t}^{2} P_{0}}$

$N_{\mathrm{t}}$ is regarded as a constant in the derivation of Eqns. 3 and 4, because in routine practice the cell numbers in each culture are closely monitored. A similar but more cumbersome derivation for a variance of mutant frequency was reported by Furth et al. [12] for a human lymphoblastoid cell line. In that paper, normality was assumed when constructing confidence intervals for the mutant frequency.

The variance of $\hat{\mu}$ should not be confused with the variance of mutant colonies, $\operatorname{var}(y)$, in parallel cultures. $\operatorname{Var}(\hat{\mu})$ is a measure of the variation of $\hat{\mu}$ obtained from a given combination of $C$ and $N_{\mathrm{t}}$ if the experiment is repeated many times.

That $\mu$ estimated from $\hat{P}_{0}$ is biased is somewhat surprising. Armitage [2] warned that some of the methods for the calculation of $\mu$ in fluctuation analysis might be biased, but the warning has been generally overlooked and the extend of bias has never been evaluated.

The bias of $\hat{\mu}$ is in general 2 orders of magnitude smaller tham $\hat{\mu}$; its exact size varies depending on the various combinations of $N_{\mathrm{t}}$ and $C$ used in the experiment (see Table 1). Because $P_{0}=\mathrm{e}^{-\mu N_{1}}$, however, a larger $N_{1}$ will increase the bias slightly (see Table 1). The mean squared error (MSE), $E(\hat{\mu}-\mu)^{2}$, a measure of deviation of $\hat{\mu}$ from $\mu$, is equal to:

$\operatorname{var}(\hat{\mu})+(\text { bias })^{2}$

The size of MSE can be minimized by maximizing both $N_{\mathrm{t}}$ and $C$, but $N_{\mathrm{t}}$ has a bigger impact.

Since $\hat{\mu}$ is biased, the $95 \%$ confidence interval of $\mu$ cannot be constructed directly. However, based on $P_{0}$, the approximate upper and lower bounds of $\mu$ can be derived by taking the natural logarithm of the $95 \%$ confidence interval of $P_{0}$, then dividing the interval by $N_{\mathrm{t}}$. The $95 \%$ confidence interval of $P_{0}$ can be obtained by using the figure for the confidence limits for proportions from statistics textbooks such as ref. 5.

As a numerical example to illustrate the effect of $N_{\mathrm{t}}$ and $C$ on the recognition of mutations and the reliability of $\hat{\mu}$, consider a hypothetical series of experiments in which $C$ is set to be $25,50,100$ or 150 , and $N_{t}$ is set to be $10^{5}, 10^{6}$, or $10^{7}$. The expected results are shown in Table 1. There are 4 combinations of $N_{\mathrm{t}}$ and $C$ in which the ratios $(R)$ of the upper and lower bounds of $\mu$ are greater than 220 , indicating that $\hat{\mu}$ values are expected to vary greatly. When $N_{t}$ is $10^{6}$ and $C$ is 50 , the 
热

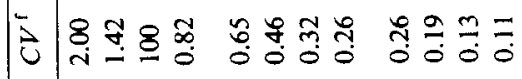

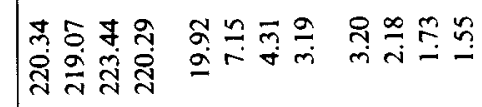

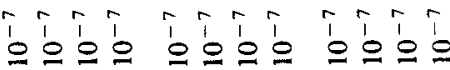
저 $\bar{x} \bar{x} \bar{x} \bar{x}$ x $\bar{x} \bar{x}$

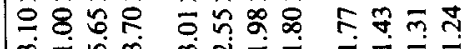
$\overbrace{1}^{1}=$

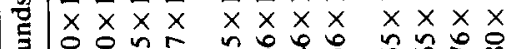

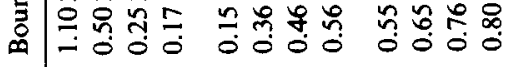

空

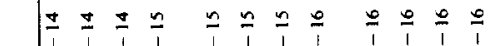

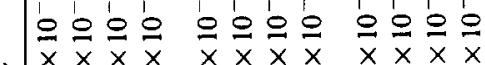

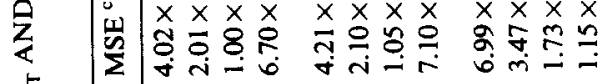
岁



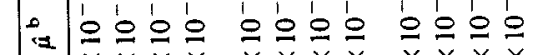



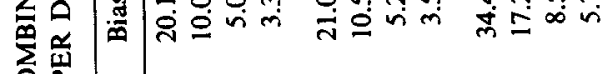
잉

它岂

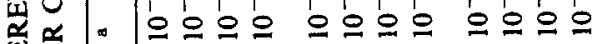
売崫

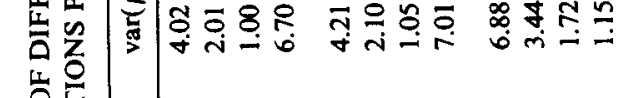


discrepancy of $\hat{\mu}$ from different experiments may reach 7.15 -fold. The variation decreases to a range of 3.19-fold when $C$ increases to 150 . When $N_{\mathrm{t}}$ is $10^{7}$, the variation of $\hat{\mu}$ becomes less dependent on the size of $C$, and the expected total number of mutations in the experiment $\left(C \mu N_{\mathrm{t}}\right)$ is much higher than those at the lower levels of $N_{\mathrm{t}}$. The results clearly demonstrate the combined influence of $N_{\mathrm{t}}$ and $C$, with $N_{\mathrm{t}}$ playing the major role, on the reliability of $\hat{\mu}$ in an experiment.

\section{Toward a reliable estimation of $\mu$}

One measure of precision in the etimate of $\mu$ is the coefficient of variation $(C V)$ :

$$
C V=\frac{\sqrt{\operatorname{var}(\hat{\mu})}}{\hat{\mu}}
$$

When $C V$ is 0.20 , the variation of $\hat{\mu}$ from experiment to experiment will be less than 3-fold (Table 1).

At an early stage in designing a fluctuation experiment, the number of sib cultures $(C)$ should be determined. From Eqns. 4 and 5 and a preliminary estimate of $\hat{\mu}_{0}$,

$$
C=\frac{1-P_{0}}{\left(\hat{\mu}_{0} N_{\mathrm{t}}\right)^{2} \cdot P_{0} \cdot(C V)^{2}}
$$

If $C V$ is set to be 0.20 , then $C=25\left(1-P_{0}\right) /\left(\hat{\mu}_{0} N_{t}\right)^{2} P_{0}$. Thus, if $\hat{\mu}_{0}=1 \times 10^{-7}$ and $N_{\mathrm{t}}=1 \times 10^{7}$, then $C$ should be set at 43 to estimate $\mu$ within a range of 2 - or 3 -fold.

For a fluctuation analysis to yield a reliable and reproducible $\hat{\mu}$, the following steps are recommended: (i) predict in advance the order of magnitude of $\mu$ to be determined, either from past experience or from the literature, (ii) use Eqn. 1 to choose a value of $N_{\mathrm{t}}$ so as to obtain an appropriate $P_{0}$, perhaps $N_{\mathrm{t}}$ equal to $0.5 \mu$, (iii) choose a $C V$ for an permissible size of variation, and (iv) use Eqn. 6 to compute $C$. The judicious choice of a combination of $N_{1}$ and $C$ will also depend on the laboratory facilities and its available resources.

\section{Effects of $N_{t}$ and $C$ on the estimation of $\mu$}

The results of 5 Expts. on spontaneous mutations from sensitivity to resistance to 6-thioguanine (6-TG) in Chinese hamster V79 cells are summarized in Table 2. The experiments were initiated with several large batches of parallel cell cultures, each being allowed to grow for different lengths of time in order to reach different final cell numbers. Some of the experiments were deliberately made less than ideal, with the intention of demonstrating the differential effect of $N_{\mathrm{t}}$ and $C$ on the reliability of estimation of $\mu$. Mutation rate is expressed as the numbers of mutations per cell per division [19,22]. The ratio of the variance to the mean of the number of resistant colonies, $\operatorname{var}(y) / \bar{y}$, is much greater in the fluctuation experiments than in the control, consistent with the notion that 6-TG resistance occurred randonly during population growth in the absence of 6-TG. When both $N_{\mathrm{t}}$ and $C$ were set small (Expt. 2), no mutation was recovered in any single culture. Mutants became detectable as $C$ was increased (Expt. 1). The large $C V$ of Expt. 1 suggests that a large 


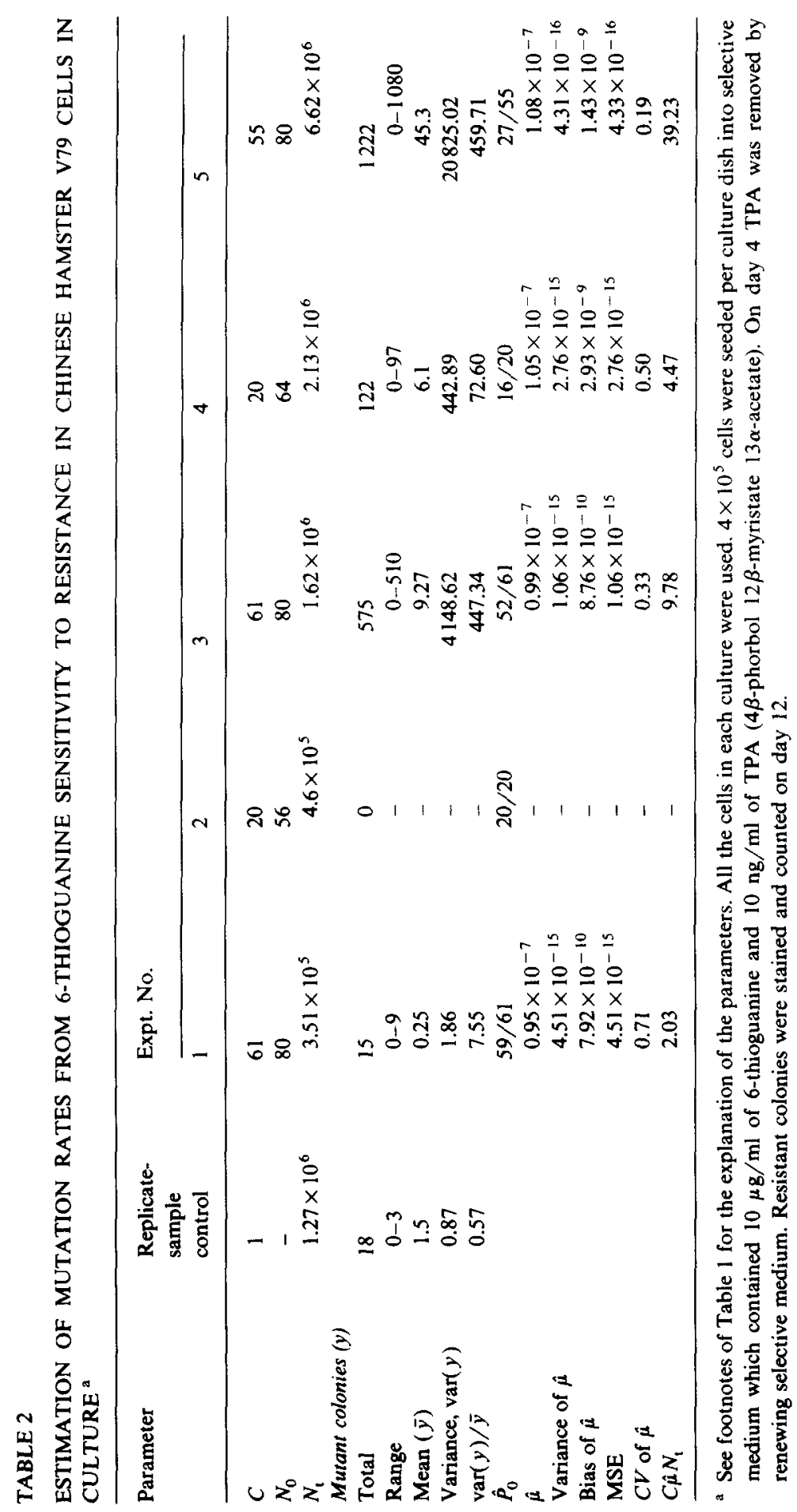


variation of $\hat{\mu}$ among repeated experiments is expected to occur, including zero estimates. As $N_{\mathrm{t}}$ was increased (Expts. 1, 3 and 5) and $C$ kept at a constant level, the sizes of $C \hat{\mu} N_{\mathrm{t}}$ increased accordingly while $\hat{P}_{0}$ and $C V$ decreased; more mutations were detected and $\hat{\mu}$ became less variable. In Expts. 3 and 4 , the sizes of $N_{\mathrm{t}}$ were similar, but Expt. 4 had a larger $C V$ because its $C$ was smaller. In Expt. 5, the value of $C V$ was 0.19 , suggesting that its $\hat{\mu}$ was reliable within a factor of 3 .

Applying the same analysis to some of the published data $[1,4,9,20,25,26]$, we find that most of the experimental designs are less than ideal. Values of $C V$ range from 0.81 to 0.22 ; most of the $C$ values used are less than ideal to ensure a narrow range of $\hat{\mu}$. The stipulation of $C V \leq 0.2$ may seem too stringent a condition for experimental design, but it seems to us that without a reproducible estimate of $\mu$, the whole exercise is useless.

Equality test of $\mu$ derived from different cell lines

To assess the biological meaning of the variation of $\hat{\mu}$ obtained from different cell lines, it is necessary to perform tests on the homogeneity of population $\mu$ values inferred from the $P_{0}$ method. Assuming that the $N_{\mathrm{t}}$ values from 2 Expts. are the same and that $\mu_{1}=\mu_{2}$, then $\mu_{1} N_{t 1}=\mu_{2} N_{t 2}$, or $P_{01}=P_{02}$. Therefore, to test the null hypothesis $\mathrm{H}_{0}: \mu_{1}=\mu_{2}$ is the same as to test $\mathrm{H}_{0}: P_{01}=P_{02}$ with $N_{\mathrm{t} 1}=N_{\mathrm{t} 2}$. Assuming that the differences of the two proportions are normally distributed, if the statistic

$$
Z=\frac{P_{01}-P_{02}}{\sqrt{\left(\frac{C_{1} P_{01}+C_{2} P_{02}}{C_{1}+C_{2}}\right)\left(1-\frac{C_{1} P_{01}+C_{2} P_{02}}{C_{1}+C_{2}}\right)\left(\frac{1}{C_{1}}+\frac{1}{C_{2}}\right)}}
$$

gives $|Z| \geq 1.96$, then $\mathrm{H}_{0}$ should be rejected under a 2-tailed test at the $5 \%$ level. An alternative test for the equality of two proportions is the exact test at the 0.05 level, based on the hypogeometric distribution. However, the computation is rather tedious for large values of $C_{1}$ and $C_{2}$.

In Table 3, equality tests on the $\mu$ values inferred from $\hat{P}_{0}$ from the literature and some of our own results are presented. Since the criterion for using the $Z$ statistic is to have the same $N_{t}$, only those experiments with similar $N_{t}$ were analyzed. For this reason and because not all $\hat{P}_{0}$ values are available, none of the published data on 'mutator' cell lines $[20,25,26]$ can be tested in this fashion. Our $Z$ test is sensitive enough to reject $\mathrm{H}_{0}: \mu_{1}=\mu_{2}$ when $\hat{\mu}_{1} / \hat{\mu}_{2}$ is as small as 3.05 (Expt. A). When the $\mu$ ratio is as small as 1.35 (Expt. D), however, $\mathrm{H}_{0}$ is accepted.

\section{Discussion}

By the application of simple statistical procedures, we have provided solutions for two long-standing and perplexing problems in quantitative mutagenesis research, viz.: the reliable estimation of mutation rate $(\mu)$ and the comparison of $\mu$ obtained from different cell lines. The first problem is especially important for mammalian 
TABLE 3

EQUALITY TEST OF MUTATION RATE $(\mu)$ INFERRED FROM THE $P_{0}$ METHOD

\begin{tabular}{|c|c|c|c|c|}
\hline \multirow[t]{4}{*}{ Parameter } & \multicolumn{4}{|l|}{ Marker } \\
\hline & \multicolumn{4}{|c|}{ 6-Thioguanine resistance ${ }^{a}$} \\
\hline & \multicolumn{2}{|l|}{ Expt. [ref.] } & & \\
\hline & \multicolumn{2}{|l|}{ A [8] } & \multicolumn{2}{|l|}{$\mathrm{B}[17,18]$} \\
\hline Cell line ${ }^{b}$ & V79 WT & V79, CTPS46 & V79 WT & $\mathrm{V} 79, \mathrm{AD}^{\mathrm{r}} 4-2$ \\
\hline$N_{0}$ & 80 & 115 & 1 & 1 \\
\hline$N_{1}$ & $1.62 \times 10^{6}$ & $1.70 \times 10^{6}$ & $3.0 \times 10^{6}$ & $3.0 \times 10^{6}$ \\
\hline$C$ & 61 & 20 & 54 & 26 \\
\hline$\hat{P}_{0}$ & $52 / 61$ & $12 / 20$ & $49 / 54$ & $10 / 26$ \\
\hline$\hat{\mu}$ & $0.99 \times 10^{-7}$ & $3.01 \times 10^{-7}$ & $3.14 \times 10^{-8}$ & $3.08 \times 10^{-7}$ \\
\hline$\hat{\mu}_{1} / \hat{\mu}_{2}$ & \multicolumn{2}{|c|}{3.05} & \multicolumn{2}{|c|}{9.80} \\
\hline$Z$ & \multicolumn{2}{|c|}{3.36} & \multicolumn{2}{|c|}{5.00} \\
\hline $\mathrm{H}_{0}: \mu_{1}=\mu_{2}$ & \multicolumn{2}{|c|}{ rejected } & \multicolumn{2}{|c|}{ rejected } \\
\hline
\end{tabular}

a 6-TG at $10 \mu \mathrm{g} / \mathrm{ml}$ in Expts. A and B; ouabain at 0.5 and $3 \mathrm{mM}$, respectively, in Expts. C and D.

somatic cells in culture, as compared to bacteria, not for cenceptual but for technical and economic reasons.

The usual problem of unreliable estimation of $\mu$ stems mainly from the absence of an appropriate set of criteria for experimental design. Through our derivation of $\operatorname{var}(\hat{\mu})$, the design problem becomes definitive and amenable to analysis. Our study suggests the necessity of using larger $N_{\mathrm{t}}$ and $C$ for a successful fluctuation analysis than are usually employed. There is a large impact of each mutational event on the estimation of $\mu$ when only a small fraction of the $C$ cultures exhibited mutations, e.g., 2 out of 120 cultures. The extent of this impact decreases as $P_{0}$ decreases because $N_{\mathrm{t}}$ increases. $C$ influences the accuracy of $\hat{\mu}$ by controlling the size of the bias as well as the $C V$. Once $N_{t}$ is decided, $C$ can be calculated before performing the experiment by using Eqn. 6 to confine $\hat{\mu}$ within a certain range of variation. $\hat{\mu}$, as inferred from $\hat{P}_{0}$, is biased. If the experiment is properly designed, however, this bias can be minimized. In that case, the deviation of $\hat{\mu}$ from $\mu$ will be too small to be of any practical significance for the accuracy of $\hat{\mu}$. The maximum likelihood method is a good choice for $\hat{\mu}$ calculation, although it does not necessarily yield an unbiased estimate $[2,3,13,15]$.

By proposing a test statistic, we have placed the comparison of $\mu$ values on a statistical basis. Lacking a proper test statistic, a mutator effect has traditionally been invoked whenever the $\hat{\mu}$ ratio between two cell lines is larger than either 5 or 10 . With the availability of our $Z$ statistic, a possible mutator effect can be detected with greater power. Our results (Table 3) suggest that cell lines CTPS 46 (Expt. A) and 


\begin{tabular}{|c|c|c|c|}
\hline \multicolumn{4}{|c|}{ Ouabain resistance $^{a}$} \\
\hline \multicolumn{4}{|l|}{ Expt. [ref.] } \\
\hline \multicolumn{2}{|l|}{$C[8]$} & \multicolumn{2}{|l|}{$\mathrm{D}[4]$} \\
\hline V79, CTPS42 & V79 CTPS43 & Mouse L cells & Mouse L cells \\
\hline 60 & 90 & 100 & 100 \\
\hline $4.6 \times 10^{6}$ & $4.0 \times 10^{6}$ & $3.0 \times 10^{7}$ & $3.1 \times 10^{7}$ \\
\hline 20 & 24 & 28 & 32 \\
\hline $2 / 20$ & $2 / 24$ & $6 / 28$ & $4 / 32$ \\
\hline \multirow[t]{4}{*}{$4.94 \times 10^{-7}$} & $6.21 \times 10^{-7}$ & $5.13 \times 10^{-8}$ & $6.93 \times 10^{-8}$ \\
\hline & & & \\
\hline & & & \\
\hline & & & \\
\hline
\end{tabular}

b CTPS 42, 43, and 46 are mutant cell lines of Chinese hamster V79 cells with an altered structure of CTP synthetase [7,8]. $\mathrm{AD}^{\mathrm{r}} 4-2$ is a V79 mutant cell line resistant to aphidicolin and has altered DNA polymerase $\alpha[6,18]$.

$\mathrm{AD}^{\mathrm{r}}$ 4-2 (Expt. B) may be more mutable than their parental lines.

The derivation of the statistic is based on the notion of comparing two proportions, $P_{01}$ vs. $P_{02}$, providing that the $N_{1}$ values from 2 Expts. are equal. This will, however, present some operational difficulties, and it is the principal drawback of our testing procedure. One solution is to monitor the population growth carefully and to stop the growth when $N_{\mathrm{t}}$ reaches a desired cell number. Because the $Z$ statistic is sensitive (Table 3 ), an equality test should be performed only when the estimation of $\mu$ is reliable (a $C V \leq 0.2$ ). We appreciate the experimental difficulties and economic constraints involved in making $C V$ smaller than 0.2 , but if instead one follows the multiple replating technique of Stocker [24], a much larger experimental scale is needed to obtain a rate estimate. Furthermore, the rate estimate so obtained is only an approximation [3], and the labor involved would be prohibitive.

\section{Acknowledgements}

The authors thank Drs. Peter Smouse, Sue-chen Ho Wu, Terence Featherstone, James Neel and Patricia Moll for helpful comments on the manuscript.

This research was supported by grant CA 26803 from the U.S. Public Health Service. 


\section{References}

1 Albertini, R.J., and R. DeMars, Somatic cell mutation detections and quantification of X-ray-induced mutations in cultured human deploid human fibrolblasts, Mutation Res., 18 (1973) 199-224.

2 Armitage, P., The statistical theory of bacterial populations subject to mutation, J. Roy. Statist. Soc. B, 14 (1952) 1-40.

3 Armitage, P., Statistical concepts in the theory of bacterial mutation, J. Hyg., 51 (1953) 162-184.

4 Baker, R.M., D.M. Brunette, R. Mankovitz, L.H. Thompson, G.F. Whitmore, L. Siminovitch and J.E. Hill, Ouabain-resistant mutants of mouse and hamster cells in culture, Cell, 1 (1974) 9-12.

5 Beyer, W.H. (Ed.), Handbook of Tables for Probability anf Statistics, The Chemical Rubber Company, Akron, OH, 1966, p. 188.

6 Chang, C.C., J.A. Boezi, S.T. Warren, C.L.K. Sabourin, P.K. Liu, L. Glatzer and J.E. Trosko, Isolation and characterization of a UV-sensitive hypermutable aphidicolin-resistant Chinese hamster cell line, Somat. Cell Genet., 7 (1981) 235-254.

7 Chu, E.H.Y., J. McLaren, I.-C. Li and B. Lamb, Pleiotropic CTP Synthetase mutants of Chinese hamster cells, Genetics, 100 (1982) s12-13.

8 Chu, E.H.Y., J. McLaren, I.-C. Li and B. Lamb, Unpublished data.

9 DeMars, R., and K.R. Held, The spontaneous azaguanine-resistant mutants of diploid human fibroblasts, Humangenetik, 16 (1972) 87-110.

10 Elmer, E., and J.C. Barrett, Measurement of spontaneous mutation rates at the $\mathrm{Na}^{+} / \mathrm{K}^{+}$ATPase locus (ouabain resistance) of human fibroblasts using improved growth conditions, Mutation Res., 97 (1982) 393-404.

$11 \mathrm{Fu}$, J., 1.C. Li and E.H.Y. Chu, The parameters for quantitative analysis of mutation rates with cultured mammalian somatic cells, Mutation Res., 105 (1982) 363-370.

12 Furth, E.E., W.G. Thilly, B.W. Peman, H.L. Liber and W.M. Rand, Quantitative assay for mutation in diploid human lymphoblasts using microtiter plates, Anal. Biochem., 110 (1981) 1-8.

13 Koch, A., Mutation and growth rates from Luria-Delbrück fluctuation tests, Mutation Res., 95 (1982) 129-143.

14 Kondo, S., A theoretical study on spontaneous mutation rate, Mutation Res., 14 (1972) 365-374.

15 Lea, D.A., and C.A. Coulson, The distribution of the numbers of mutants in bacterial populations, J. Genet., 49 (1949) 264-285.

$16 \mathrm{Li}$, I.-C., J. Fu, Y.-T. Hung and E.H.Y. Chu, The reliability of mutation rates estimates by fluctuation analysis in cultured mammalian cells: Effect of the number of parallel cultures and the final population size on the variance of the rate estimates, Genetics, 100 (1982) s40-41.

17 Liu, P.K., Isolation and characterization of an aphidicolin resistant mutator mutant of Chinese hamster cells, Ph.D., dissertation, Michigan State University, 1981.

18 Liu, P.K., C.-C. Chang, J.E. Trosko, D.K. Dube, G.M. Martin and L.A. Loeb, Mammalian mutator mutant with an aphidicolin-resistant DNA polymerase $\alpha$, Proc. Natl. Acad. Sci. (U.S.A.), 80 (1983) 797-801.

19 Luria, S.E., and M. Delbrück, Mutations of bacteria from virus sensitivity to virus resistance, Genetics, 28 (1943) 491-511.

20 Meuth, M., N. L'Heureux-Huard and M. Trudel, Characterization of a mutator gene in Chinese hamster ovary cells, Proc. Natl. Acad. Sci. (U.S.A.), 76 (1979) 6505-6509.

21 Morrow, J., On the relationship between spontaneous mutation rates in vivo and in vitro, Mutation Res., 33 (1975) 367-372.

22 Newcombe, H.B., Delayed phenotypic expression of spontaneous mutation in Escherichia coli, Genetics, 33 (1948) 447-476.

23 Shapiro, A., The kinetics of growth and mutation in bacteria, Cold Spring Harbor Symp. Quant. Biol., 11 (1946) 228-234.

24 Stocker, B.A.D., Measurement of rate of mutation of flagellar antigenic phage in Salmonella typhimurium, J. Hyg., 47 (1949) 398-413.

25 Warren, S.T., R.A. Schultz, C.C. Chang, M.H. Wade and J.E. Trosko, Elevated spontaneous mutation rate in Bloom syndrome fibroblasts, Proc. Natl. Acad. Sci. (U.S.A.), 78 (1981) 3133-3137.

26 Weinberg, G., B. Ullman and D.W., Martin, Mutator phenotypes in mammalian cell mutants with distinct biochemical defects and abnormal deoxyribonucleotide triphosphate pools, Proc. Natl. Acad. Sci. (U.S.A.), 78 (1981) 2447-2451. 